\title{
Silicon based electron, optical and X-ray imagers
}

\begin{abstract}
Charge-Coupled Devices (CCDs) have been invented almost 40 years ago. [1] Several years later CCDs overtook film as the media for scientific optical imaging. Since two decades the CCDs have created and have dominated the market for electronic cameras and camcorders. A few years later complementary metal oxide semiconductor (CMOS) imaging detectors have been introduced and made their move into the charge-coupled device (CCD) optical market. Low cost, low power, on-chip system integration, highspeed operation and tolerance to high-energy radiation sources are unique features that make CMOS detectors popular. The huge commercial interest of optical imager is responsible for remarkable continuous improvements in both technologies and it is hard to know which technology will dominate the optical market a few years from now (2009).
\end{abstract}

The continuous development of optical imagers is having impact in scientific application of imaging of electrons in electron microscopy and X-ray imagers. Traditionally the image of electron microscope was viewed by direct projection onto a fluorescent viewing screen coated with a phosphor or scintillator material such as zinc sulfide. The image could be photographically recorded by exposing a photographic film or plate directly to the electron beam. More recent electron detectors have phosphor coupled by means of a lens optical system or a fibre optics light-guide to the optical CCD. The image detected by the CCD may be displayed on a monitor or computer. This is an example of an indirect detection of electrons by silicon. First a part of electron energy was converted into the energy of optical photons which were then detected in an optical imager (CCD). In this proces the linearity of the signal at the output of CCDs is propotional the the total energy of electrons impinging on the screen, however, the sharpness of the picture is degrated when compare to the direct record on the film. The time to read a single frame of CCD is a large fraction of a second. Many important studies require measurements on the $\mathrm{ms}\left(10^{-3}\right.$ second $)$ scale. One can be tempted to conclude that a simple substitution of CCD with a CMOS imager would provide a panacea. For several applications this simple substitution can lead to a desirable performance when the read-out speed is of primary importance. The detectors for the most demanding applications of electron microscopy should provide "perfect" imaging information with a millisecond read-out speed. The imaging information contains the sharpness express in modulation transfer functions (MTF), linearity and the modulation transfer functions and the dynamic range. It is likely that silicon as a direct detection medium may fulfill the most stringent requirements of imaging for the electron microscopy.

The situation is similar, however, not identical with imaging of X-rays for crystallography and similar applications. The direct detection of electron in silicon provides charge signal which is more than one order of magnitude larger than the signal of the indirect method where the scintillation photons are recorded. There is no dispersion of light and the sharpness of the image may by only dependent on the number of pixels in the silicon detector. Physics of the detection process in silicon has to be taken into 
account to make the full usage of all pixels. Figure 1 shows a simulation of trajectories (in projection) of 200 electrons $200 \mathrm{keV}$ each right after reaching the entrance side of a silicon detector.

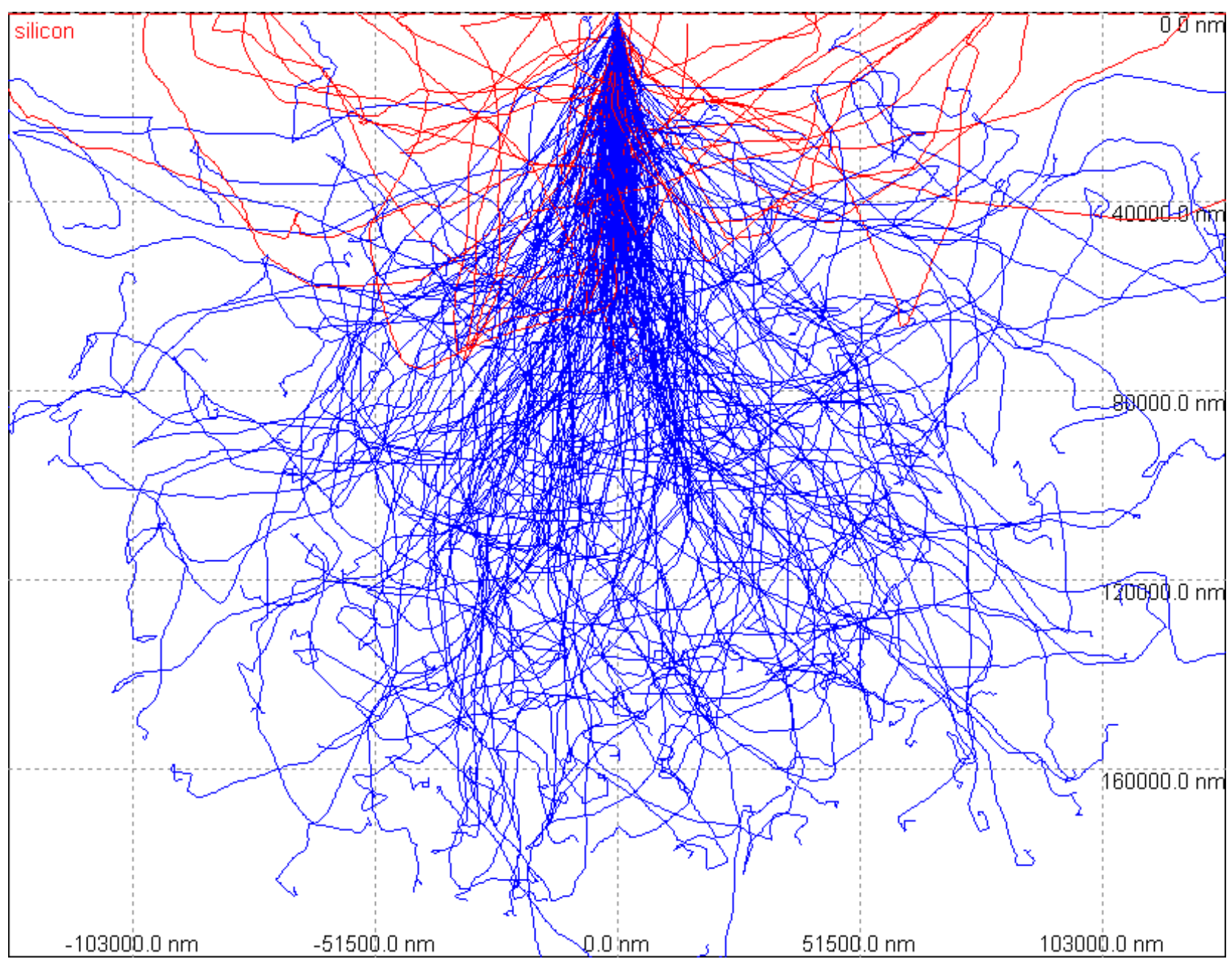

Figure 1. 200 trajectories of $200 \mathrm{keV}$ electrons on 400 um silicon

Due to the multiple scattering of electrons in silicon the center of gravity of the charge produced by electrons of the microscope does not give the position of the incident electron. In the talk the possible method to limit negative effects of the multiple scattering will be discussed. Differences with the problems of light and X-ray detection will be listed. 